\title{
Width of Sunspot Generating Zone and Reconstruction of Butterfly Diagram
}

\author{
V. G. Ivanov ${ }^{1,2}$ and E. V. Miletsky ${ }^{1}$ \\ ${ }^{1}$ Central Astronomical Observatory at Pulkovo, Saint-Petersburg, Russia \\ 2 email: ivanov. vg@gao. spb.ru
}

\begin{abstract}
Based on the extended Greenwich-NOAA/USAF catalogue of sunspot groups it is demonstrated that the parameters describing the latitudinal width of the sunspot generating zone (SGZ) are closely related to the current level of solar activity, and the growth of the activity leads to the expansion of SGZ. The ratio of the sunspot number to the width of SGZ shows saturation at a certain level of the sunspot number, and above this level the increase of the activity takes place mostly due to the expansion of SGZ. It is shown that the mean latitudes of sunspots can be reconstructed from the amplitudes of solar activity. Using the obtained relations and the group sunspot numbers by Hoyt and Schatten (1998), the latitude distribution of sunspot groups ("the Maunder butterfly diagram") for the 18th and the first half of the 19th centuries is reconstructed and compared with historical sunspot observations.
\end{abstract}

Keywords: Solar cycle, observations; Sunspots, statistics

\section{Introduction}

The 11-year cycle of solar activity, which is now referred to as "the SchwabeWolf law" after the names of its discoverers (Schwabe, 1843, 1844; Wolf, 1852), was found in the study of sunspot number variations in time. Later a gradual shift of the mean latitude of the sunspot generating zone (SGZ) to the solar equator in the course of the 11-year cycle was found (Carrington, 1858; Spörer, 1874). This spatial regularity of the cycle is now called "the Spörer law". The famous "butterfly diagram", first drawn by Maunder (1904), can be regarded as a graphic illustration of this law.

Since sunspots are among the most prominent manifestations of the solar magnetic field, these two laws, apparently, reflect different aspects of the solar magnetic cycle. Therefore, for better understanding of the physical nature of the 11-year cycle it is very important to explore the relations between the latitudinal distribution of sunspots and solar activity.

A close connection is known between the sunspot activity indices (in particular, the Wolf number $\mathrm{W}$ ) at the maximum of a solar cycle with such characteristics as the sunspot latitudes averaged over the year of the solar maximum (Waldmeier, 1955) or over the solar cycle (Li et al., 2003). Recently Miletskii and Ivanov (2009, hereafter referred to as Paper I) confirmed this connection by reproducing the Waldmeier relation with new data on sunspot activity obtained after 1955. In investigation of statistical 
characteristics of the latitudinal distribution of sunspots in different cycles it was also found that the mean solar latitude of a given cycle and their latitudinal dispersion are highly correlated (Solanki, Wenzler, and Schmitt, 2008). Therefore, latitudinal characteristics of the 11-year cycles taken as a whole are related closely to the power of these cycles.

It is interesting to look for the relation between the latitudinal sunspot distribution and the instantaneous level of solar activity. One of such possible candidates is the latitudinal width of SGZ. It was found in the 1950s by Becker (1954) and Gleissberg (1958) that a quantity which they introduced as a measure of the SGZ width depends upon the phase of the 11-year cycle and its largest values fall on the epoch of the maximum of the cycle. However, they did not find any dependence of this quantity upon either the magnitude of the 11-year cycle or the current level of solar activity.

In Paper I we found that the yearly means of the SGZ width (SZW), expressed as the difference of maximal and minimal latitudes of sunspot groups, are tightly related to the level of sunspot activity characterized by the Wolf numbers. In the present paper we continue this study, considering other characteristics of SZW and sunspot activity indices and using both yearly and solar rotation means of the corresponding values. We will also demonstrate that behavior of the activity can be used to find the mean latitude of the SGZ. Using the obtained regularities we will develop an approach which allows us to reconstruct the latitudinal parameters of sunspot distribution (and, thereby, to restore the butterfly diagram) before the middle of the 19th century on the basis of the available information about the amplitudes of the activity.

\section{Indices of the Number and Latitudinal Distribution of Sunspots}

\subsection{DATA AND INDICES}

Using the data on sunspot latitudes from the Greenwich catalogue and its NOAA/ USAF extension ${ }^{1}$ for the epoch 1874-2006, we calculate daily values of several sunspot indices. In particular, we obtain the daily numbers of sunspot groups $(\mathrm{G})$, total sunspot areas (SA), and the mean latitudes of sunspots weighted with the sunspot area (LA). For the same data we find the standard deviations of sunspot group latitudes $(\sigma)$ and the highest $(\mathrm{LAH})$ and lowest (LAL) latitudes of sunspots for a given day. When we calculate the latter quantities, we consider the highest latitude to be equal to the lowest if there is only one sunspot group in a given hemisphere.

${ }^{1}$ http $: / /$ solarscience.msfc.nasa.gov/greenwch.shtml 


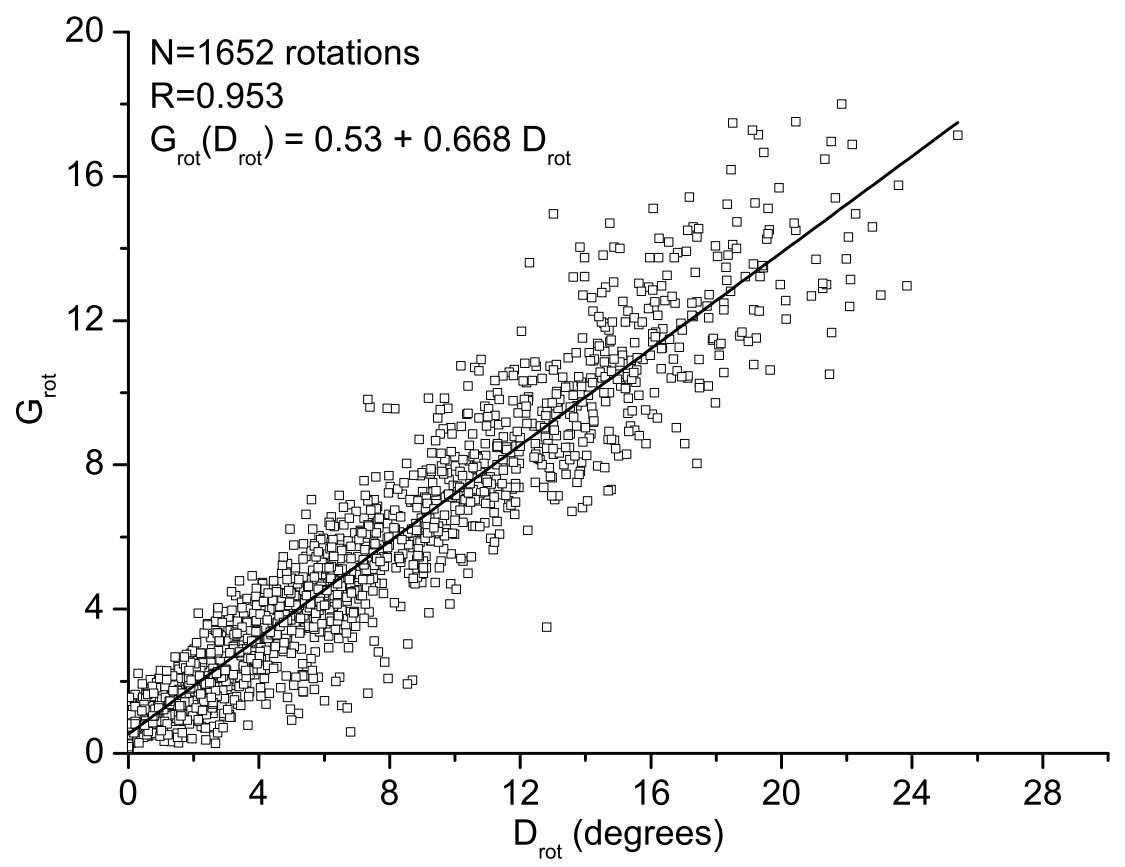

Figure 1. The relation between the rotation means of the sunspot group index $\mathrm{G}_{\text {rot }}$ and the measure of the SGZ width $\mathrm{D}_{\text {rot }}$.

In Paper I we used indices LAH and LAL as characteristics of the upper and lower boundaries of SGZ, and the derived index D = LAL - LAH (the difference between the highest and lowest latitudes, i.e. the latitudinal size of the wings of the butterfly diagram) as a measure of SZW. In the present paper we use also another possible measures of SZW: the daily standard deviations of sunspot latitudes $\sigma$ and the extent of the zone $\Delta \phi_{\rho}$ on a given level of the sunspot density $\rho$ (which will be discussed in Sec. 3.1).

In the following we use yearly and solar rotation means of indices, so that the above mentioned daily values will be averaged over the corresponding time ranges.

\subsection{Relations Between the Amplitude and Latitudinal Indices of Sunspots}

In the interval 1874-2006 (133 years) the yearly indices of sunspot activity (the Wolf number W, sunspot group index G, and their total area SA) are proved to be strongly correlated to all the aforenamed measures of SZW 
Table I. Correlations between the yearly means of sunspot activity indices and various measures of SZW.

\begin{tabular}{|c|c|c|c|c|c|c|c|c|c|}
\hline & $\mathrm{G}$ & $\mathrm{W}$ & $\mathrm{SA}$ & $\mathrm{D}$ & $\mathrm{D}^{2}$ & $\sigma$ & $\sigma^{2}$ & $\Delta \phi_{0.03}$ & $\Delta \phi_{0.03}^{2}$ \\
\hline G & & 0.982 & 0.971 & 0.989 & 0.944 & 0.920 & 0.933 & 0.936 & 0.970 \\
\hline $\mathrm{W}$ & & & 0.985 & 0.975 & 0.944 & 0.912 & 0.936 & 0.914 & 0.955 \\
\hline SA & & & & 0.958 & 0.926 & 0.889 & 0.913 & 0.898 & 0.938 \\
\hline D & & & & & 0.956 & 0.949 & 0.965 & 0.943 & 0.980 \\
\hline $\mathrm{D}^{2}$ & & & & & & 0.858 & 0.936 & 0.845 & 0.928 \\
\hline$\sigma$ & & & & & & & 0.969 & 0.944 & 0.946 \\
\hline$\sigma^{2}$ & & & & & & & & 0.904 & 0.947 \\
\hline$\Delta \phi_{0.03}$ & & & & & & & & & 0.979 \\
\hline
\end{tabular}

both for global and hemispheric values of these indices (see Table I). In particular, as it was shown in Paper I, the correlation coefficient $R(\mathrm{~W}, \mathrm{D})=$ 0.975 . It is remarkable that the maximum value in Table $\mathrm{I} R(\mathrm{G}, \mathrm{D})=0.989$ corresponds to the correlation between an activity amplitude index and a latitudinal index, whereas the highest correlation within the group of activity amplitude indices (G, W, SA), which are commonly believed to be highly correlated, is lower $(R(\mathrm{~W}, \mathrm{SA})=0.985)$. One should note that for 133 points for which the correlations were calculated, their confidence probabilities (for $R=0.989$ ) are over three standard deviations. We can see also that the relations between alternative measures of SZW are high enough, the highest being between $D$ and $\Delta \phi^{2}$.

To examine how the relation between sunspot activity and SZW depends on the range of averaging, we calculated the correlation between solar rotation means $G_{\text {rot }}$ and $D_{\text {rot }}$ of the indices $G$ and $D$ for the same epoch 1874-2006 (1652 solar rotations). One can see in Figure 1 that the obtained dependence can be fairly well presented by the linear relation

$$
\mathrm{G}_{\text {rot }}=0.53+0.668 \mathrm{D}_{\text {rot }} \quad(R=0.953)
$$

and has high consistency which follows from uniform distribution of points over the ranges of the indices. A decrease in the correlation as compared with the value obtained for yearly means of the indices is as small as 0.036 and the mean square error between the observed and model series is $\delta\left(\mathrm{G}_{\text {rot }}\right)=1.18$.

The behavior of sunspot indices and SZW taken separately in different hemispheres are also proved to be in good agreement. For the yearly means of indices $\mathrm{G}$ and $\mathrm{D}$ in the northern $(\mathrm{N})$ and southern $(\mathrm{S})$ hemispheres one can obtain the following relations:

$$
\mathrm{GN}(\mathrm{DN})=0.052+0.355 \mathrm{DN} \quad(R(\mathrm{DN}, \mathrm{GN})=0.980, \quad \delta(\mathrm{GN})=0.39)
$$




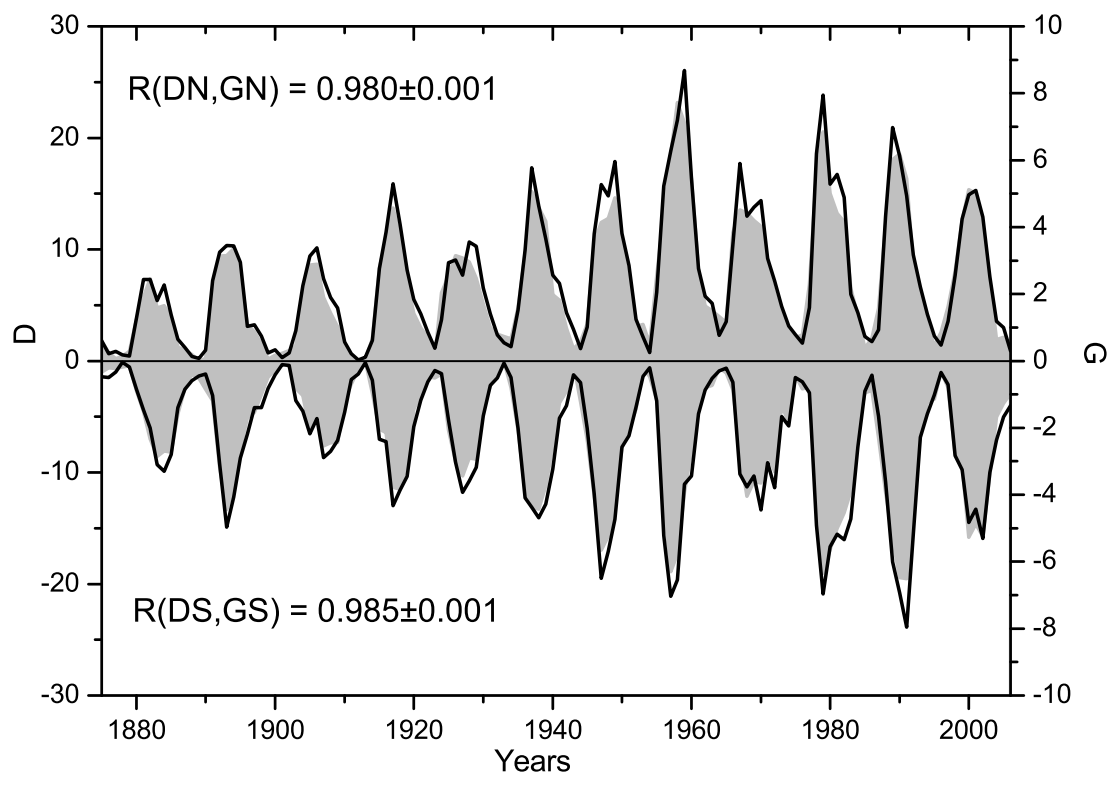

Figure 2. Hemisphere components of indices G and D. The series GN (solid line) and DN (dotted line), corresponding to the northern hemisphere, are plotted to the positive direction of the ordinate, and GS and DS (corresponding to the southern hemisphere) to the negative direction.

and

$$
\mathrm{GS}(\mathrm{DS})=-0.001+0.360 \mathrm{DS} \quad(R(\mathrm{DS}, \mathrm{GS})=0.985, \quad \delta(\mathrm{GS})=0.33) .
$$

In Figure 2 the hemispheric indices are presented. The series GN and DN are shown by the solid and dotted lines, respectively, and plotted to the positive direction of the ordinate. Similarly, GS and DS are plotted to the negative direction of the ordinate. Some disagreements between the behavior of indices $\mathrm{G}$ and $\mathrm{D}$ take place only during the minima of 11-year cycles, but in other epochs of the cycles the indices agree very well.

Recently (Paper I) we showed that the yearly means of indices D and $\mathrm{W}$ are closely related. The above examination proves that this regularity holds for other characteristics of SZW and activity indices, and for different ranges of averaging. Therefore, the growth of solar activity is accompanied by an increase of SZW. Using this relation and having the latitudes of sunspots (and SZW), one is able to make a reliable estimation of the level of solar activity. Conversely, by knowing this level, one can calculate rather 


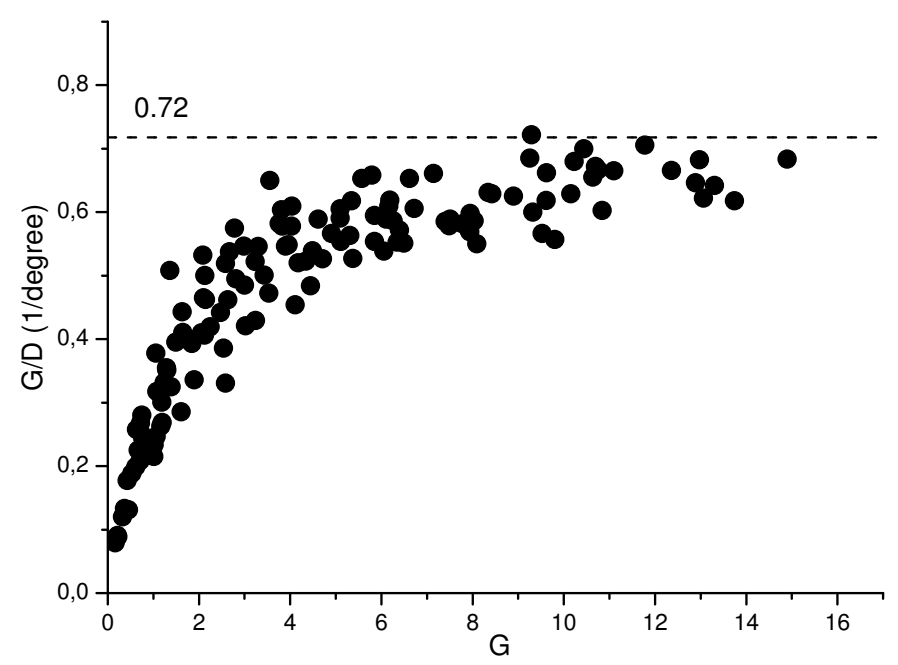

Figure 3. The dependence of the G/D ratio upon G (yearly means). The dashed line corresponds to the saturation level 0.72 .

accurately the current extent of "the wings of Maunder's butterfly" (see Section 3).

\subsection{Saturation Level of G/D}

The ratio $G / D$ of the sunspot group index $(G)$ to the width of the corresponding zone (D) can be, under certain conditions, treated as a mean density of the latitudinal distribution of sunspots. In Figure 3 the dependence of this value upon $G$ is plotted. We can see that, as $G$ increases, a linear growth of the ratio $G / D$ slows down, and saturates at a certain level $(\approx 0.72)$.

A comparison of the behavior of the yearly means of G/D (see Figure 4) shows that, in each 11-year cycle, this value starts increasing in the minimum and reaches a level which is nearly the same for all 11-year cycles. A further increase of the activity takes place mostly due to the widening of SGZ in latitude. Therefore, there is a limitation of the mean latitudinal density of sunspots.

\section{Reconstruction of the Butterfly Diagram}

Systematic data on the coordinates of sunspot groups are available in the extended Greenwich catalogue since 1874. Earlier information on the spatial distribution of sunspots is scarce, however. For $1854-1873$ the mean latitudes of sunspots can be obtained by the compilation of pre-Greenwich 


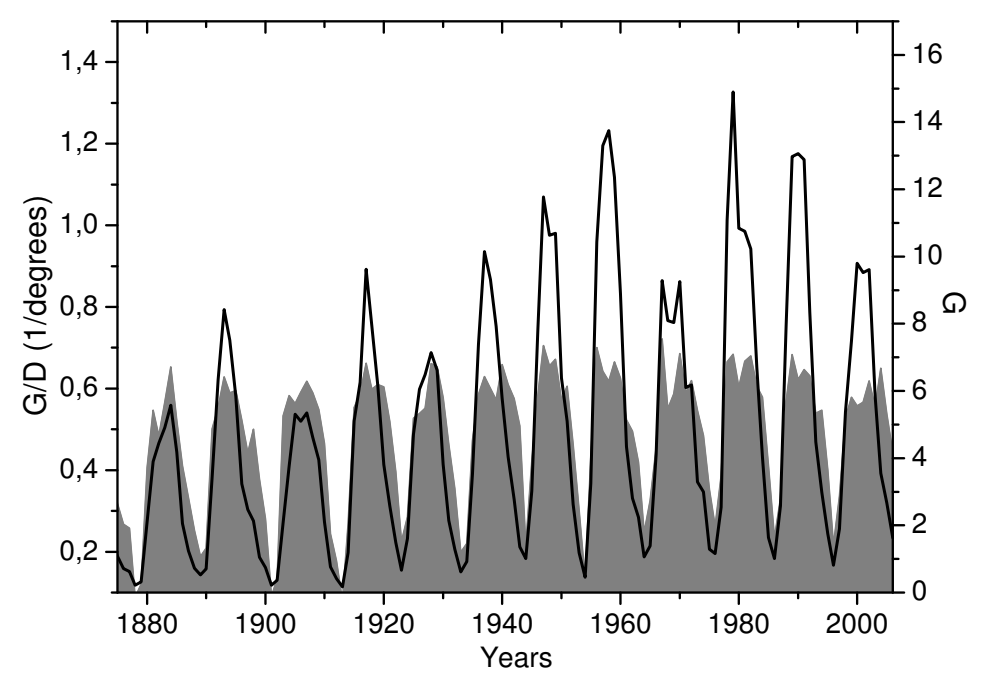

Figure 4. The yearly means of the ratio G/D (the dotted line) and the group number index G (the solid line).

observations (Nagovitsyn et al., 2004) ${ }^{2}$. But before the middle of the 19th century we have only non-systematic drawing of the Sun (e.g., Ribes and Nesme-Ribes, 1993; Arlt, 2008, 2009).

The relation between the amplitude of solar activity and SZW found above can help us to reconstruct the latitudinal distribution of sunspots in the pre-Greenwich epoch from some proxy data. We will use as such proxy the group sunspot numbers (GSN), which were calculated by Hoyt and Schatten (1998) since 1610. Its correlation with the sunspot group index $\mathrm{G}$ on the overlapping period of data is higher than 0.99 for yearly means, and the linear relation

$$
\mathrm{G} \approx \mathrm{GSN} / 11.76
$$

is valid for these two indices. Therefore, in the following we will not make a difference between GSN (rescaled according to Equation (1)) and G.

\subsection{Parametrization of the Butterfly Diagram}

In order to reconstruct the spatial distribution of sunspots from a scalar index, one first should select a proper parametrization of the distribution. The latitudinal distribution of sunspot groups in the butterfly diagram for a given year in a given hemisphere can be approximately described, e.g., by the mean latitude of sunspots $\phi_{0}$ and some measure of SZW. As the latter, it is possible to use any of the SZW indices mentioned in Section 2.1, but for our purpose it is convenient to use the extents of the zone $\Delta \phi_{\rho}$.

\footnotetext{
${ }^{2}$ See ESAI database: http://www.gao.spb.ru/database/esai/
} 


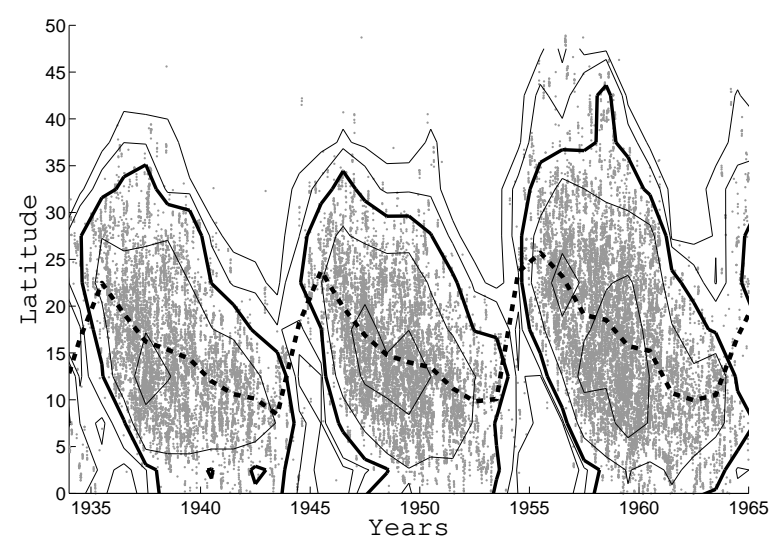

Figure 5. A portion of the butterfly diagram. The levels of the sunspot group densities are shown by solid contours, with the bold contours corresponding to the density level $\rho=0.03$ groups/degree/day. The dashed line shows the mean latitude of sunspot groups.

To determine it we plot the contours of latitudinal sunspot group densities on the butterfly diagram, and select a "representative" contour which outlines the wing of the butterflies fairly well (see Figure 5). In the following we select $\rho=0.03$ groups/degrees/day (the thick line in Figure 5) and will omit the index $\rho$ on $\Delta \phi_{\rho}$. The upper $\phi_{\text {up }}$ and lower $\phi_{\text {low }}$ borders of the wing in a given hemispheres are determined as the yearly averages of latitudes of the corresponding contours, and the half-widths of the wing in the hemisphere, as $\Delta \phi_{\text {up }}=\phi_{\text {up }}-\phi_{0}$ and $\Delta \phi_{\text {low }}=\phi_{0}-\phi_{\text {low }}$.

Common sunspot activity indices, such as GSN, which we use for the reconstruction of the sunspot distribution, do not include explicit information of the north-south asymmetry of sunspots. Therefore, we will neglect the asymmetry, coming to the values of these parameters averaged over two hemispheres.

Of course, in case of need, by selecting an additional level of the density $\rho^{\prime}$, one can obtain the corresponding indices $\phi_{\text {up,low }}^{\prime}$ and parametrize the latitudinal distribution of sunspots in more detail.

Therefore, we have selected three yearly series $\left(\phi_{0}, \Delta \phi_{\text {up }}\right.$, and $\left.\Delta \phi_{\text {low }}\right)$, which describe the form of the butterfly diagram (see Figure 6 ), and will look for their relations to the level of solar activity described by $\mathrm{G}$.

\subsection{Reconstruction of the Half-Widths of the Butterfly DIAGRAM}

The average half-width of the butterfly wings $\Delta \phi=\left(\Delta \phi_{\text {up }}+\Delta \phi_{\text {low }}\right) / 2$ can be used as a measure of the latitudinal extent of SGZ. Table I shows that its 


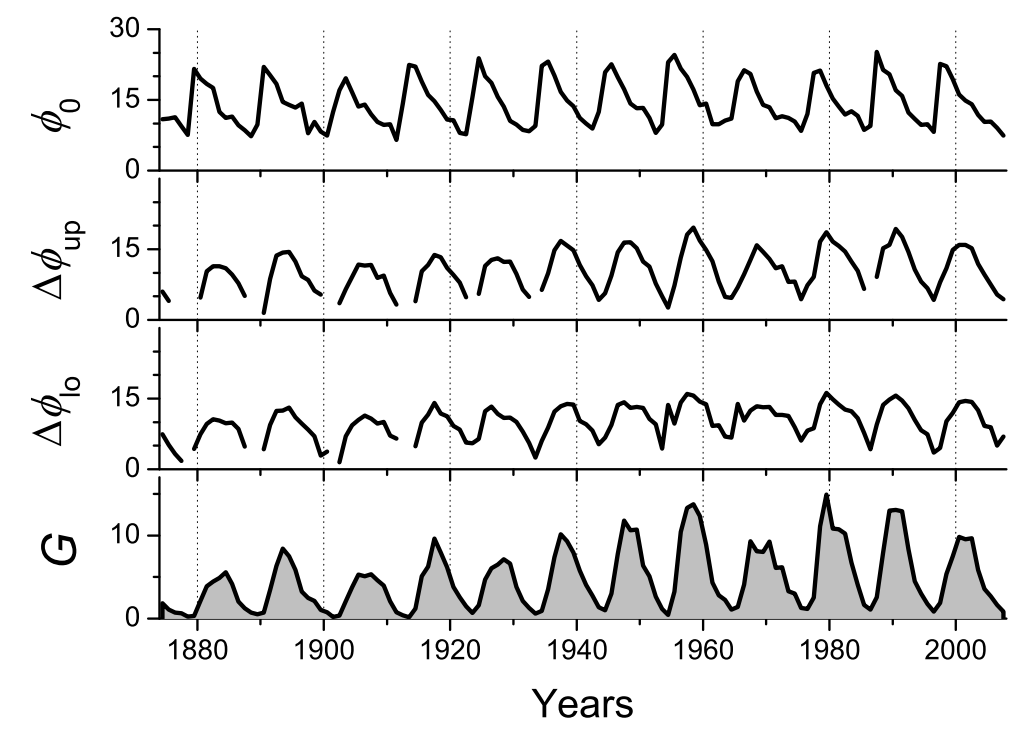

Figure 6. From top to bottom: the mean latitudes of sunspot groups $\phi_{0}$, the half-widths of the butterfly diagram $\Delta \phi_{\text {up }}$ and $\Delta \phi_{\text {low }}$, and the number of sunspot groups $\mathrm{G}$.
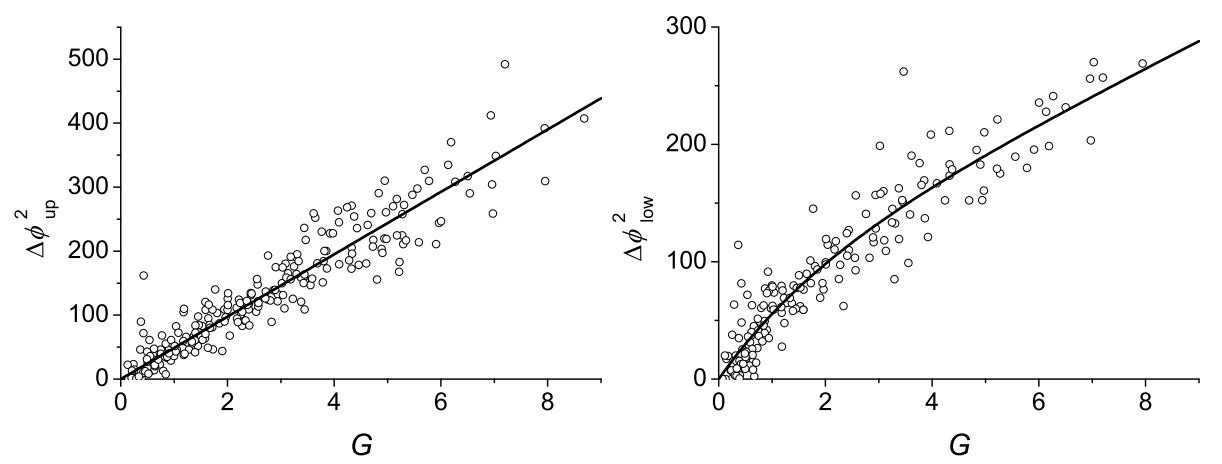

Figure 7. The relationship between the number of sunspot groups $\mathrm{G}$ and the half-widths of the butterfly diagram $\Delta \phi_{\text {up }}$ and $\Delta \phi_{\text {low }}$.

square $\Delta \phi^{2}$ is in good correlation with G. However, to improve the accuracy it is useful to treat $\Delta \phi_{\text {up }}$ and $\Delta \phi_{\text {low }}$ separately. In Figure 7 one can see that their dependence upon $\sqrt{\mathrm{G}}$ is close to linear and can be described by the regression equations

$$
\Delta \phi_{\mathrm{up}}(\mathrm{G})=\alpha \sqrt{\mathrm{G}}
$$

and

$$
\Delta \phi_{\text {low }}(\mathrm{G})=\beta \sqrt{\mathrm{G}+\gamma(1-\exp (-\mathrm{G} / 2))}
$$

where

$$
\alpha=6.98, \quad \beta=4.79, \quad \gamma=3.61
$$




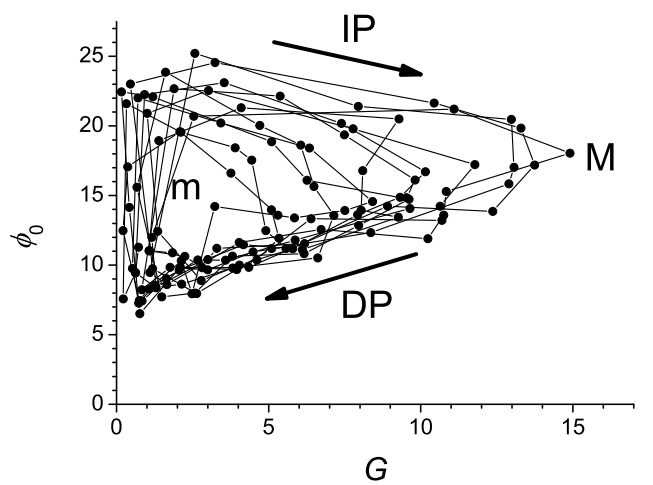

Figure 8. The relations between the yearly index G and the mean latitude of sunspots $\phi_{0}$ at various phases of the 11-year cycle: the minimum $(\mathrm{m})$, increasing phase (IP), maximum $(\mathrm{M})$, and decreasing phase (DP).

The correlation coefficients for both regressions are 0.94 and the standard errors are about $1.5^{\circ}$. We introduced a week non-linear tuning by parameter $\gamma$ in the second relation to improve the fitting at low G.

\subsection{Reconstruction of the Mean Latitudes of Sunspots}

The reconstruction of the mean sunspot latitudes is a more difficult problem, since there is no univocal relationship between this index and solar activity indices. Figure 8 shows that the relation between the mean sunspot latitude and $\mathrm{G}$ differs on different phases of the 11-year solar cycle. Therefore, in order to find $\phi_{0}(t)$ for a given year $t$, one should take into account not only $\mathrm{G}(t)$, but also the time derivatives of this index (or equivalently, $\mathrm{G}\left(t^{\prime}\right)$ for $\left.t^{\prime} \neq t\right)$.

Nagovitsyn (2008) proposed a method for the reconstruction of the mean latitudes which is based on the mapping of an original characteristic of solar activity $X$ into a pseudo-phase space

$X(t) \longrightarrow \Psi(t)=[X(t-n \cdot \Delta), X(t-(n-1) \cdot \Delta), \ldots, X(t), \ldots, X(t+n \cdot \Delta)]$

and search for a linear regression which links $\Psi$ and the required index $\phi_{0}$. Using this method, he obtained a reconstruction of the mean latitudes of sunspots since 1621 .

However, in this approach it is difficult to estimate the errors of the model. Below we apply another method, which does not assume linear relationships. For the reconstruction we use an artificial two-layers feed-forward neural network (see, e.g., Conway (1998) and references therein) with sigmoid (linear) transfer functions of the first (second) layer, respectively. The input variable of the neural network (NN) is the group index $\mathrm{G}$ mapped into the 


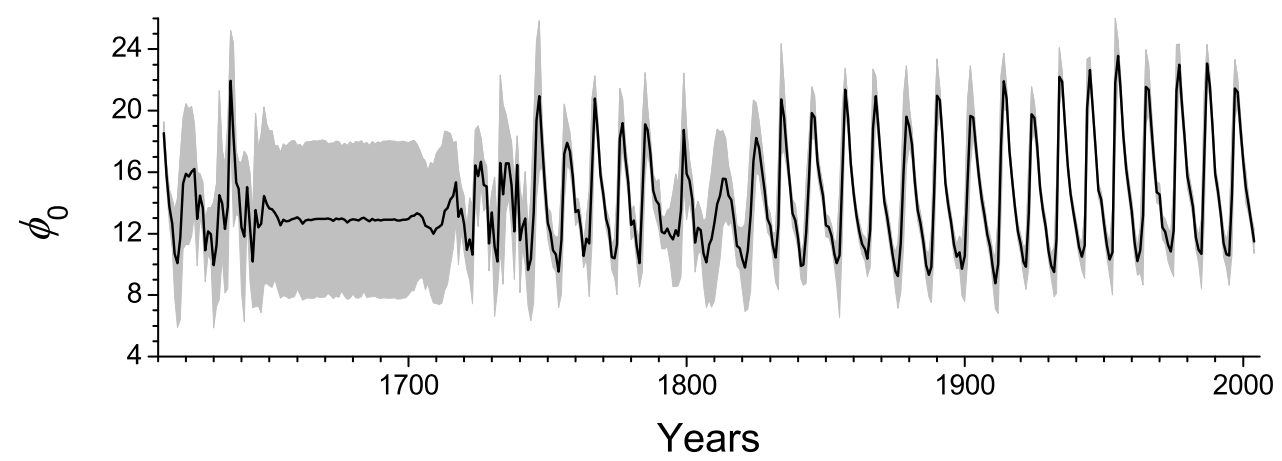

Figure 9. The reconstruction of the mean sunspot latitudes $\phi_{0}(1612-2004)$ by the NN model. The gray halftone corresponds to the error of reconstruction $\delta \phi_{0}$.

pseudo-phase space by Equation (4) (with $\Delta=1$ year) and the output variable is a 20-dimensional vector which is made of the yearly means of sunspot group densities in the five-degree latitudinal intervals $\left[-50^{\circ},-45^{\circ}\right]$, $\ldots,\left[+45^{\circ},+50^{\circ}\right]$. We are free to vary three parameters of the model, namely the dimension of the input vector $2 n+1$, the number of neurons of the first hidden layer $h$, and the initial state of NN.

Selecting a random set of these parameters (under the condition $2 \leq$ $n \leq 23$ and $2 \leq h \leq 11$ ) and training each of the corresponding NN, we obtain an ensemble of $Q$ different models. Then we use as the input of these models GSN by Hoyt and Schatten (1998) recalculated to the G scale by Equation (1) and mapped into the pseudo-phase space. For each of the resulting output density distributions we calculate a series of mean sunspot latitudes $\phi_{0, i}, i=1, \ldots, Q$. Finally, the resulting reconstruction of $\phi_{0}$ (Figure 9) is obtained by averaging these series over the ensemble, and the corresponding standard deviation $\delta \phi_{0}$ can be treated as an estimate of the error in the method. A comparison between the mean latitudes obtained by Nagovitsyn (2008) and our reconstruction shows that the series are in fair agreement. However, the errors in our reconstruction for low levels of the global solar activity are significantly larger. It can be explained by the fact that, in the epoch of the Maunder minimum, the system was located in a domain of the pseudo-phase space which differs from the one used for the training of NN. Therefore, the mean latitude can be more or less reliably reconstructed by this method only after the beginning of the 18th century.

\subsection{Results of Reconstruction}

The reconstructed parameters $\phi_{0}, \phi_{\text {up }}$, and $\phi_{\text {low }}$ can be used to restore the form of the butterfly diagram in the 18th and the first half of the 19th centuries (Figure 10). 

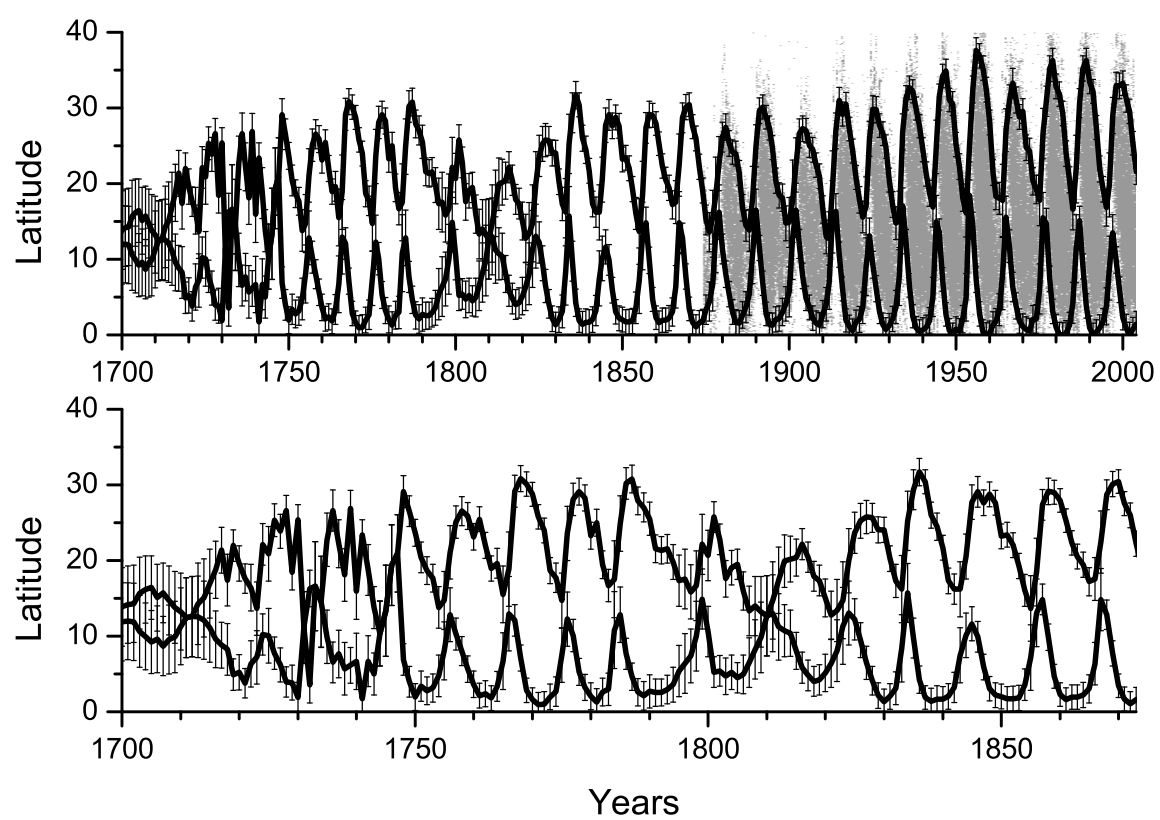

Figure 10. The reconstruction of the northern wing of the butterfly diagram. The full series for 1700-2004 years is shown on the top panel, and the reconstruction for 1700-1874 on the bottom panel. The vertical bars correspond to the errors of the reconstruction. The gray halftone on the top panel is the observed sunspot distribution.

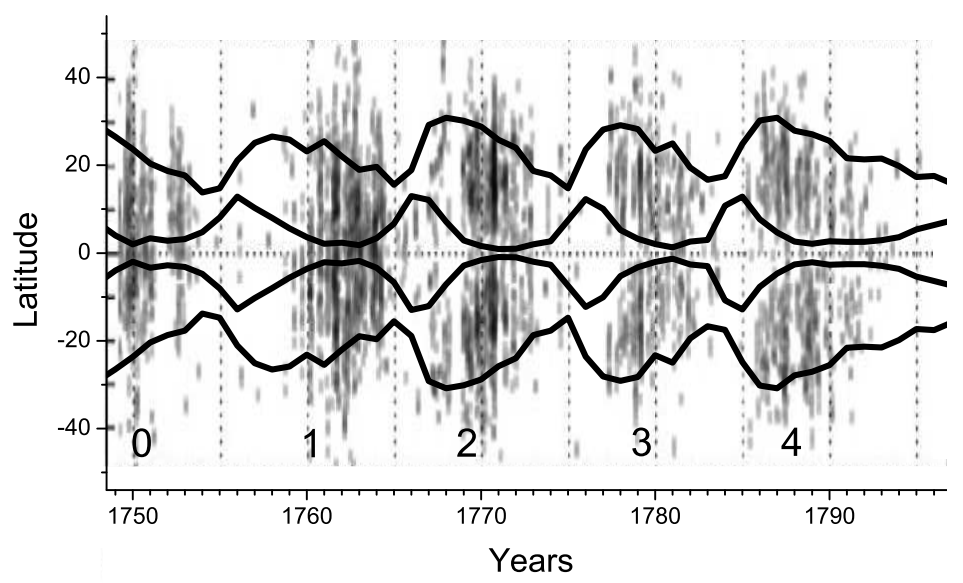

Figure 11. A portion of our reconstruction (the bold lines correspond to $\phi_{\text {up }}$ and $\phi_{\text {low }}$ ) superposed on the distribution of sunspots by Staudacher (Arlt, 2009, Figure 2a). The numerals below are the cycle numbers. 
It is interesting to compare the obtained diagram with observations. Unfortunately, the largest set of sunspot drawings made in Paris Observatory (Ribes and Nesme-Ribes, 1993) was collected in the earlier epoch (16601719). Another long series of sunspot coordinates was recently extracted from semi-centennial (1749-1796) observations of a German amateur astronomer Staudacher (Arlt, 2009). In Figure 11 the butterfly diagram by the data of Staudacher (the upper panel of Figure 2 from Arlt (2009), which contains 6285 sunspot positions) is compared with $\Delta \phi_{\text {up }}$ and $\Delta \phi_{\text {low }}$ of our reconstruction. One can see that for cycles 3 and 4 the distributions are more or less in agreement. For the earlier three cycles the form and size of the wings visibly differ from our reconstruction. However, as it is noted by Arlt (2009), the distributions in cycles 0-2 have some anomalies: the excess of sunspots near the solar equator, unclear equatorward migration of sunspots during the cycle, etc. It is not clear whether these features correspond to a real behavior of the Sun or it is an artifact caused by methods of observation or data processing. The data of Staudacher are not uniformly distributed over time (during cycles 0-3 observations are more frequent than later). Besides, possibly, the data are affected by "the factor of attention", i.e. more frequent observations in days with larger numbers of sunspots as compared with days with few or no sunspots (for 1016 days with observations there are only 17 days without sunspots). This factor would obviously lead to systematic overestimation of SZW.

Another possible reason for the peculiar form of cycles 0-3, as it was also mentioned by Arlt (2009), is the dominance of the quadrupolar mode of the magnetic field in this epoch. Of course, this effect cannot be reproduced by our model, which is built under the assumption of the dipolar butterfly-like form of the sunspot distribution.

Taking into account possibility of such effects, we consider the agreement of these two distributions to be satisfactory.

\section{Conclusions}

In this paper we continued the investigation of Paper I on the relationship between the level of solar activity and some characteristics of the latitudinal distributions of sunspots. In particular, we showed that in the 11-year cycle the characteristic width of the sunspot generating zone is tightly related to the level of solar activity, and this relation holds for different indices of sunspot activity, regardless of the selection of parameters to define the extent of latitudinal sunspot distribution or various scales of averaging.

We found that a certain saturation level exists for the ratio $(G / D)$ of the sunspot number index $(G)$ to the latitudinal size of the corresponding zone (D). Above this level, the increase of the activity takes place mostly due to 
the expansion of SGZ. In all the explored sunspot cycles the ratio reaches this level and, therefore, practically does not depend upon the amplitudes of the cycles.

We also showed that the mean latitude of the sunspot distribution is related to the levels of the activity in the given sunspot cycle

Using the obtained relation we reconstructed the form of the latitudinal distribution of sunspots ("the Maunder butterfly diagram") in the epochs where little or no direct observations of spatial sunspot distribution are available. The reconstruction is rather accurate for middle or high levels of global solar activity (1720-1863), but during the epochs of grand minima (in the Maunder minimum) its results are not reliable.

Our results can be regarded as an additional confirmation of complementarity of spatial and amplitude characteristics of solar magnetic fields. The obtained regularities can be used as diagnostic criteria for the choice of adequate models of the solar cyclicity.

\section{Acknowledgements}

This work is supported in part by the grant of Russian Foundation for Basic Research No. 10-02-00391 and the grant "Leading Scientific Schools"

No. 3645.2010.2. The authors are also grateful to Yu. Nagovitsyn for useful discussions.

\section{References}

Arlt, R.: 2008, Solar Phys. 247, 399.

Arlt, R.: 2009, Solar Phys. 255, 143.

Becker, U.: 1954, Z. Astrophys. 35, 137.

Carrington, R.C.: 1858, Mon. Not. Roy. Astron. Soc. 19, 1.

Conway, A.J.: 1998, New Astron. Rev. 42, 343.

Gleissberg, W.: 1958, Z. Astrophys. 46, 219.

Hoyt, D.V., Schatten, K. H.: 1998, Solar Phys. 179, 189.

Li, K.J., Wang, J.X., Zhan, L.S., Yun, H.S., Liang, H.F., Zhao, H.J., Gu, X.M.: 2003, Solar Phys. 215, 99.

Maunder, E.W.: 1904, Mon. Not. Roy. Astron. Soc. 64, 747.

Miletskii, E.V., Ivanov, V.G.: 2009, Astron. Rep. 53, 857. (Paper I).

Nagovitsyn, Yu.A.: 2008, Astrophys. Bull. 63, 43.

Nagovitsyn, Yu.A., Ivanov, V.G., Miletsky, E.V., Volobuev, D.M.: 2004. Solar Phys., 224, 103.

Ribes, J.C., Nesme-Ribes, E.: 1993, Astron. Astrophys. 276, 549.

Schwabe, H.: 1843, Astron. Nachr. 20, 283.

Schwabe, H.: 1844, Astron. Nachr. 21, 233.

Solanki, S.K., Wenzler, T., Schmitt, D.: 2008, Astron. Astrophys. 483, 623.

Spörer, G.: 1874, Beobachtungen der Sonnenflecken zu Anclam, W. Engelmann, Leipzig. 
Waldmeier, M.: 1955, Ergebnisse und Probleme der Sonnenforschung, Geest \& Portig, Leipzig.

Wolf, R.: 1852, Comptes Rendus Acad. Sci. 35, 704. 
Ivanov_Miletsky-arxiv.tex; 15/08/2018; 5:11; p.16 